\title{
Classical Stabilization of Homogeneous Extra Dimensions
}

\author{
Sean M. Carroll, ${ }^{*}$ James Geddes,${ }^{\dagger}$ Mark B. Hoffman,${ }^{\ddagger}$ and Robert M. Wald ${ }^{\S}$ \\ Enrico Fermi Institute and Department of Physics, \\ University of Chicago \\ 5640 S. Ellis Avenue, Chicago, IL 60637, USA \\ hep-th/0110149. EFI-2001-38
}

October 22, 2018

\begin{abstract}
If spacetime possesses extra dimensions of size and curvature radii much larger than the Planck or string scales, the dynamics of these extra dimensions should be governed by classical general relativity. We argue that in general relativity, it is nontrivial to obtain solutions where the extra dimensions are static and are dynamically stable to small perturbations. We also illustrate that intuition on equilibrium and stability built up from non-gravitational physics can be highly misleading. For all static, homogeneous solutions satisfying the null energy condition, we show that the Ricci curvature of space must be nonnegative in all directions. Much of our analysis focuses on a class of spacetime models where space consists of a product of homogeneous and isotropic geometries. A dimensional reduction of these models is performed, and their stability to perturbations that preserve the spatial symmetries is analyzed. We conclude that the only physically realistic examples of classically stabilized large extra dimensions are those in which the extra-dimensional manifold is positively curved.
\end{abstract}

\footnotetext{
*carroll@theory.uchicago.edu

$\dagger$ jm-geddes@uchicago.edu

${ }_{\ddagger}^{\ddagger} \mathrm{mb}$-hoffman@uchicago.edu

${ }^{\S}$ rmwa@midway.uchicago.edu
} 


\section{Introduction}

The idea that spacetime may have more than 4 dimensions has had a long and distinguished history. In the context of string theory or other quantum theories of gravity, the extra dimensions typically would be expected to be of a size set by the string or Planck length. In such cases, one would not expect a classical description of the extra dimensions to be valid. However, in recent years, the possibility has been raised that "large" extra dimensions may be present, and a number of such explicit models have been proposed ([1]; see [2] for a review, and [3] for recent experimental limits). It seems reasonable to expect that classical general relativity should be adequate to analyze the equilibrium and stability of these models.

In general relativity, it is nontrivial to obtain models with static extra dimensions, and it is even more nontrivial to stabilize these extra dimensions with respect to small perturbations. Furthermore, intuition developed from non-gravitational physics with regard to equilibrium and stability can be highly misleading. Indeed, the difficulties in obtaining stable compact dimensions and the counter-intuitive behavior of many models are well illustrated by a simple example.

Consider a static, flat, $n$-torus universe. (In this example, the $n$-torus will be taken to be all of space, but a similar example could be given where the $n$-torus comprises only the extra dimensions.) This spacetime is, of course, a static, equilibrium solution of the vacuum Einstein equation. Is this solution stable? The following argument suggests that it should not be: There is no scale that sets the values of the "moduli" of the torus. In particular, there is an equilibrium solution for all possible values of the radii of the torus. Therefore, at best, one would expect the torus to be neutrally (un)stable: If one of the dimensions of the torus is perturbed toward collapse, there should be nothing to prevent that dimension from collapsing to zero size in a finite time. It is not difficult to prove that this expectation is correct: There exist exact solutions of the vacuum Einstein's equation corresponding to a linear decrease (or growth) of the size of one of the dimensions of the torus with time, so an arbitrarily small perturbation will cause the torus to collapse within a finite time.

\footnotetext{
${ }^{1}$ These exact solutions with linear expansion or contraction of one of the radii of the torus are actually flat. They can be obtained by starting with a wedge of $(1+1)$ dimensional Minkowski spacetime where the action of Lorentz boosts is spacelike (the "Milne universe") and making a periodic identification under Lorentz boosts.
} 
Can the above $n$-torus universe be stabilized via the addition of suitable matter fields? One might expect that it would be straightforward to do so by simply adding some mattersuch as iron struts - whose total energy was minimized at a particular chosen set of values of the radii of the torus. This matter would then set a suitable equilibrium scale for the torus, and one might expect that the $n$-torus universe at the energy minimizing radii would be a stable, equilibrium configuration. (In the somewhat different context of a single extra dimension with orbifold boundary conditions, this is essentially the idea of the GoldbergerWise stabilization mechanism [4].) However, it is easy to see that this mechanism does not work in this case. In fact, we will see from eq. (5) below that if one adds matter satisfying the strong energy condition with strict inequality holding at at least one point, then no static solutions whatsoever can exist, so all solutions must be expanding or contracting in at least one spatial dimension. Furthermore, if we consider a contracting solution with the same rate of contraction in all directions (thereby corresponding to a flat FRW solution with periodic identifications), then it also can be seen that the presence of a positive pressure enhances the rate of collapse in the sense that, with the same initial conditions, a universe filled with matter possessing positive pressure will collapse to zero size faster than a universe filled with dust. Thus, rather than stabilizing the torus, adding "iron struts" or other matter with positive pressure actually enhances the tendency to collapse.

The difficulties in achieving stable equilibrium of compact dimensions are not special to the above $n$-torus example. Indeed, a simple constraint on the existence of stationary solutions can be obtained as follows. Consider a stationary spacetime, with timelike Killing field $t^{a}$, which is spatially compact or compactifiable (i.e., possessing appropriate spatial symmetries so that the noncompact directions could be compactified via identifications). This encompasses many models considered in the literature, including all of the ones we shall consider below in this paper. Then $t^{a}$ satisfies (see, e.g., Appendix C of [5])

$$
\nabla^{a} \nabla_{a} t_{b}=-R_{b c} t^{c}
$$

Since $\nabla_{a} t_{b}=\nabla_{[a} t_{b]}$, this equation may be rewritten in differential forms notation as

$$
d * d t=-2 *(R \cdot t)
$$

where $(R \cdot t)_{a} \equiv R_{a b} t^{b}$. Integrating this equation over a compact (or compactified) spacelike 
slice $\Sigma$, we obtain

$$
\int_{\Sigma} *(R \cdot t)=0
$$

i.e.,

$$
\int_{\Sigma} R_{a b} n^{a} t^{b} d \Sigma=0
$$

where $n^{a}$ denotes the unit normal to $\Sigma$, and $d \Sigma$ denotes the volume element on $\Sigma$ induced from the spatial metric on $\Sigma$. In particular, if the spacetime is static (rather than merely stationary) and we choose $\Sigma$ to be orthogonal to $t^{a}$, then we obtain

$$
\int_{\Sigma} R_{a b} n^{a} n^{b} V d \Sigma=0
$$

where $V=\left(-t^{a} t_{a}\right)^{1 / 2}$.

Eq. (4) and/or eq. (5) provide a significant integral constraint on the existence of stationary solutions with compact or compactifiable spacelike slices. The strong energy condition is the requirement that $\left(T_{a b}-\frac{1}{2} T g_{a b}\right) v^{a} v^{b} \geq 0$ for all timelike $v^{a}$. It is violated by a positive cosmological constant and by matter fields whose stress-energy is dominated by (positive) potential energy, but is satisfied by most other forms of matter usually considered. In the presence of Einstein's equation, the strong energy condition is equivalent to the condition that $R_{a b} v^{a} v^{b} \geq 0$ for all timelike $v^{a}$. Eqs. (雨) and (5) indicate that it will be difficult to produce stationary solutions to Einstein's equation with matter without violating the strong energy condition. In particular, as already mentioned above, in the static case, if there is a point where $R_{a b} n^{a} n^{b}>0$, then there also must be a point where $R_{a b} n^{a} n^{b}<0$, thereby violating the strong energy condition.

Similar conclusions concerning the difficulties with the existence of static solutions satisfying the strong energy condition can be drawn by appealing to the singularity theorems. In particular, Theorem 9.5.4 of [5] asserts that if a spacetime possesses a compact spatial slice, if matter satisfies the strong energy condition, and if, in addition, there are no closed timelike curves and the timelike and null generic conditions hold, then the spacetime must be singular in the sense of timelike or null geodesic incompleteness. Consequently, all stationary globally hyperbolic spacetimes possessing a compact or compactifiable Cauchy surface - such as the static $n$-torus discussed above - must violate the generic condition. By the above theorem, any perturbation of such a spacetime that satisfies the generic condition must produce a singularity. 
The above arguments against the existence of stable, static solutions can be evaded by allowing for the presence of a positive cosmological constant or other forms of matter violating the strong energy condition. Even so, it is still far from obvious how to make stable, static models. Indeed, the Einstein static universe provides a good illustration of the kind of difficulties one can encounter: Although a static solution can be obtained by "balancing" a positive cosmological with an ordinary form of matter, such an equilibrium is unstable.

The main purpose of this paper is to investigate the constraints placed by classical general relativity on the existence and stability of static solutions to Einstein's equation in the case where "space" is taken to be a product of an arbitrary number of homogeneous and isotropic geometries of arbitrary curvature - in other words, conventional Kaluza-Klein theory [6]. In sections 2 and 3, we restrict attention to the case of a product of two such geometries - one of which can be taken to be ordinary, macroscopic space, and the other of which can be taken to be the "large" (compared with the Planck scale) extra dimensions. In section 2, we show that if matter satisfies the null energy condition $\left(T_{a b} l^{a} l^{b} \geq 0\right.$ for all null $\left.l^{a}\right)$, then existence of a static solution requires either that the compact space be positively curved, or that the spacetime be locally Minkowski and the stress-energy tensor vanish identically. Thus, provided only that the null energy condition holds, the extra dimensions in the static models with the symmetries we consider cannot have negative curvature, and they cannot even be flat unless the universe is completely empty. More generally, we show that for any static, homogeneous solution with matter satisfying the null energy condition, the Ricci curvature of space must always be nonnegative.

In section 3, we consider a particular model that has previously been considered in the literature [0, 8, 9, 10, 11], in which spherical extra dimensions are stabilized via a balance between vacuum energy and gauge fields (and the curvature of the internal space). We perform a dimensional reduction of it, thereby reducing it to an ordinary Robertson-Walker model with a self-interacting scalar field. The static solution is unstable in the sense that an arbitrarily small perturbation of it will result in a ("big bang") singularity. However, the perturbed model appears to be cosmologically acceptable.

Section 4 is devoted to exploring the relationship between energy minimization versus equilibrium and stability in general relativity. In non-gravitational physics, a configuration 
which minimizes the total matter energy corresponds to a stable equilibrium configuration, and stability arguments are typically made on the basis of energy minimization. However, such arguments are, at best, highly suspect in general relativity. In asymptotically flat spacetimes - where total energy is well defined - configurations of minimum total energy should correspond to stable equilibrium configurations [12], but the total energy of the spacetime is not equal to the integrated energy of matter in the spacetime, so minimization of the total matter energy may have little to do with the conditions for stable equilibrium. The situation is considerably worse in compact or compactifiable spaces, where no nontrivial notion of total energy exists. To investigate the relationship between minimization of total matter energy and stable equilibrium, we consider a Robertson-Walker spacetime filled with a fluid, and ask the following two questions: (i) What conditions on the pressure of the fluid must be satisfied in order that the total energy of the fluid be minimized? (ii) What conditions on the pressure of the fluid must be satisfied in order to have a stable, static solution to Einstein's equation? We shall see in section 4 that, in general, the conditions for equilibrium in general relativity bear no relationship with the conditions for energy extremization. Interestingly, however, in $2+1$ dimensions, it turns out that the conditions for equilibrium in general relativity actually coincide with the conditions for energy extremization. Remarkably, in this case we find that the conditions for stable equilibrium in general relativity are precisely that the matter energy be a local maximum - exactly the opposite of what would be expected in non-gravitational physics.

Finally, in section 5, we generalize the results of sections 2 and 3 to the case where the spatial geometry is a product of an arbitrary number of homogeneous and isotropic geometries. We show that, again, if matter satisfies the null energy condition, the existence of a static solution requires nonnegative curvature of each of the geometries; it is not possible to compensate for some negative curvature by the addition of positive curvature in a different factor. Following [10, 13] we also perform a dimensional reduction in this case, re-expressing the model as an ordinary Robertson-Walker cosmology with a collection of self-interacting scalar fields. Considering a specific example with four extra dimensions in the form of a product of two two-spheres [10], we investigate the cosmological acceptability of such compactifications.

Our analysis leaves numerous questions unanswered. We do not, for example, consider 
off-diagonal perturbations to our product spacetimes or perturbations that do not respect the spatial symmetries of the extra dimensions. Also, models where the standard-model fields are confined to a "brane" with a thickness much smaller than the size of the extra dimensions are incompatible with our assumption of perfect homogeneity in the extra dimensions. (In particular, Randall-Sundrum spacetimes with a single finite extra dimension [14] rely on the tension of the branes on the boundary, and are therefore not addressed by our analysis.) While a natural first step is to assume that the energy localized on the brane is smaller than that in the "bulk" and can hopefully be neglected, it is important to subsequently check that the introduction of a nonzero brane tension does not destabilize the solution. (Some progress in including the effects of nonzero brane tension has been made by considering metrics generated by global topological defects [15].)

\section{Static, homogeneous solutions in general relativity}

In this section we examine static homogeneous spacetimes, without regard to questions of stability (considered in later sections). We consider spacetimes of the form $\mathbf{R} \times \mathcal{M}_{1} \times \mathcal{M}_{2}$, where $\mathbf{R}$ is the timelike direction and $\mathcal{M}_{1}$ and $\mathcal{M}_{2}$ are maximally symmetric manifolds of dimensionality $N$ and $n$, respectively. We can think of $\mathcal{M}_{1}$ as representing the "large" spatial dimensions and $\mathcal{M}_{2}$ as representing the "extra" ones, although they will be treated on an identical footing. The metric can be written

$$
d s^{2}=-d t^{2}+g_{I J} d x^{I} d x^{J}+\gamma_{i j} d y^{i} d y^{j}
$$

where $g_{I J}\left(x^{K}\right)$ and $\gamma_{i j}\left(y^{k}\right)$ are metrics on manifolds of constant curvature (either positive, negative, or zero). Note in particular that $g_{I J}\left(x^{K}\right)$ and $\gamma_{i j}\left(y^{k}\right)$ are independent of time. Indices $I, J$ run from 1 to $N$, while $i, j$ run from 1 to $n$. We define curvature parameters by

$$
K=\frac{R\left[g_{I J}\right]}{N(N-1)}, \quad k=\frac{R\left[\gamma_{i j}\right]}{n(n-1)},
$$

where $R$ is the Ricci scalar constructed from the appropriate spatial metric.

For a stress-energy source we take a perfect fluid defined by an energy density $\rho$ and pressure in the large and small dimensions, $P$ and $p$ :

$$
T_{00}=\rho
$$




$$
\begin{aligned}
T_{I J} & =P g_{I J} \\
T_{i j} & =p \gamma_{i j} .
\end{aligned}
$$

There are three independent nontrivial components of Einstein's equation, corresponding to the $00, I J$ and ij components:

$$
\begin{aligned}
\frac{1}{2} N(N-1) K+\frac{1}{2} n(n-1) k & =8 \pi G \bar{\rho} \\
\frac{1}{2}(N-1)(N-2) K+\frac{1}{2} n(n-1) k & =-8 \pi G \bar{P} \\
\frac{1}{2} N(N-1) K+\frac{1}{2}(n-1)(n-2) k & =-8 \pi G \bar{p},
\end{aligned}
$$

where $G$ is Newton's constant (in $N+n+1$ dimensions). In (11-13) we have put overbars on the energy density and pressures to emphasize that these relations hold at a static solution.

We next impose the null energy condition $\left[\right.$ (NEC), that $T_{a b} l^{a} l^{b} \geq 0$ for all null vectors $l^{a}$. In the context of our model, the NEC is equivalent to the requirements

$$
\begin{aligned}
& \rho+P \geq 0 \\
& \rho+p \geq 0 .
\end{aligned}
$$

Taking appropriate linear combinations of (11-13) yields

$$
\begin{aligned}
8 \pi G(\bar{\rho}+\bar{P}) & =(N-1) K \\
8 \pi G(\bar{\rho}+\bar{p}) & =(n-1) k .
\end{aligned}
$$

From comparing (16-17) with (14-15), we see immediately that the curvatures $K, k$ must be either zero or positive. (The curvature cannot be made negative by choosing a onedimensional manifold, as a one-dimensional space must have zero curvature.) Thus, in a static spacetime obeying the NEC, with spatial sections described by a product of two symmetric spaces, neither spatial factor can be negatively curved. In particular, the NEC must be violated in order to consider stabilization of compact hyperbolic extra dimensions, which have recently been investigated [16].

\footnotetext{
${ }^{2}$ The NEC is in fact weaker than the "weak" energy condition, that $T_{a b} v^{a} v^{b} \geq 0$ for all timelike vectors $v^{a}$. By continuity the weak energy condition implies the NEC, but not vice-versa. In particular, the NEC permits a negative energy density if there is an equal and opposite pressure, and thus allows for a cosmological constant of either sign, while the weak energy condition prohibits any negative energy density, and thus allows only a nonnegative cosmological constant.
} 
Several possibilities remain open: both spaces may be flat, one may be flat and the other positively curved, or both may be positively curved. If both spaces are flat $(K=k=0)$, we see from (11-13) that all of the components of the stress-energy tensor must vanish, $\bar{\rho}=\bar{P}=\bar{p}=0$. This solution is (locally) empty Minkowski space.

The nonempty static solutions obeying the NEC are thus those in which at least one space is positively curved, and neither is negatively curved. This implies that toroidal compactification $(k=0)$ is only possible if the large dimensions are positively curved $(K>0)$. In fact, however, this case is not physically realistic, for two reasons. First, in the real universe the three observed spatial dimensions are flat to an excellent approximation. (Evidence for spatial flatness has been provided by measurements of the anisotropy spectrum of the cosmic microwave background [17]; for our present purposes, however, all we need to know is that radius of curvature of the large dimensions is significantly larger than any relevant microphysical scale.) Second, when (in the next section) we consider stability of a model with both flat and positively-curved dimensions, we will find that small perturbations induce oscillations in the positively curved space and a big-bang singularity in the flat dimensions. Hence, a toroidal set of extra dimensions would be unstable to collapsing to zero volume (or expanding to infinite volume). We therefore conclude that the only physically relevant examples of large extra dimensions obeying the NEC are those with positive curvature.

The above result on the non-negativity of spatial curvature for static solutions can be significantly generalized as follows. Consider any static, spatially homogeneous spacetime, with static Killing field $t^{a}$. Now, any static Killing field satisfies

$$
\nabla_{a} t_{b}=-2 V^{-1} t_{[a} \nabla_{b]} V
$$

where $V=\left(-t^{a} t_{a}\right)^{1 / 2}$. However, for a spatially homogeneous spacetime, we have $\nabla_{a} V=0$, so $t^{a}$ is covariantly constant. It then follows from eq. (囵) that $R_{a b} t^{b}=0$, where $R_{a b}$ denotes the Ricci curvature tensor of the spacetime metric. Let $s^{a}$ be any unit "spatial" vector (i.e., $t^{a} s_{a}=0$ ), and let $l^{a}=V^{-1} t^{a}+s^{a}$. Then $R_{a b} l^{a} l^{b}=R_{a b} s^{a} s^{b}$. However, since $l^{a}$ is null, by Einstein's equation, we have

$$
R_{a b} l^{a} l^{b}=8 \pi G\left(T_{a b}-\frac{1}{2} g_{a b} T\right) l^{a} l^{b}=8 \pi G T_{a b} l^{a} l^{b}
$$

Consequently, the NEC together with Einstein's equation implies $R_{a b} s^{a} s^{b} \geq 0$ for all spatial vectors $s^{a}$, with equality holding if and only if $T_{a b} l^{a} l^{b}=0$. Furthermore, since $t^{a}$ is covariantly 
constant, we have $R_{a b} s^{a} s^{b}=\tilde{R}_{a b} s^{a} s^{b}$, where $\tilde{R}_{a b}$ denotes the Ricci curvature of space. Thus, for any static, spatially homogeneous spacetime satisfying the null energy condition, the Ricci curvature of space must always be nonnegative.

\section{An example: stabilizing $S^{2}$ with gauge fields}

Having established the criteria for static solutions with a single extra-dimensional space, we now will examine in detail a specific example to understand the sense in which it can be "stable". Our example will be a spacetime with four macroscopic spacetime dimensions and two positively curved extra dimensions, stabilized by balancing a cosmological constant and a gauge field wrapped around the extra dimensions. (This mechanism was recently examined by Sundrum [11]; a nonabelian version was originally investigated by Horvath, Palla, Cremmer and Scherk [7], which was generalized to larger numbers of extra dimensions by Freund and Rubin [8], and stability was discussed by Randjbar-Daemi, Salam and Strathdee [9]. A thorough discussion, including multiple compact spaces, is given by Guenther and Zhuk [10].) We consider the conventional dimensional reduction of this theory, rewriting the six-dimensional Lagrangian as a four-dimensional Lagrangian plus a scalar field (the radion) representing the size of the extra dimensions. The effective potential for the radion will be found to have two minima: one at a finite radius, and one at infinite radius. This kind of analysis is by no means original (see for example [18] and references therein), but we will go through it carefully for pedagogical purposes.

Let $G_{a b}$ be the metric for the full spacetime with coordinates $X^{a}$, and denote by $G_{N+n+1}$ Newton's constant in the full spacetime. We consider $N$ large spatial dimensions and $n$ extra dimensions, so that indices $a, b$ run from 0 to $N+n$; we will specialize to the case $N=3$, $n=2$ after performing the dimensional reduction. We consider metrics of the form

$$
d s^{2}=G_{a b} d X^{a} d X^{b}=g_{A B}(x) d x^{A} d x^{B}+b^{2}(x) \gamma_{i j}(y) d y^{i} d y^{j}
$$

where the $x^{A}$ are coordinates in the $(N+1)$-dimensional spacetime and the $y^{i}$ are coordinates on the extra-dimensional manifold, again taken to be a maximally symmetric space with metric $\gamma_{i j}$. However, for the dimensional reduction analysis given below, it will not be assumed that the metric $g_{A B}$ of the large dimensions has any symmetries. The action is the 
$(N+n+1)$-dimensional Hilbert action, plus a matter term we leave unspecified for now:

$$
S=\int d^{N+n+1} X \sqrt{-G}\left\{\frac{1}{16 \pi G_{N+n+1}} R\left[G_{a b}\right]+\widehat{\mathcal{L}}_{M}\right\},
$$

where $\sqrt{-G}$ is minus the square root of the determinant of $G_{a b}, R\left[G_{a b}\right]$ is the Ricci scalar of $G_{a b}$, and $\widehat{\mathcal{L}}_{M}$ is the matter Lagrangian density with the metric determinant factored out.

The first step is to dimensionally reduce the action (21): we express everything in terms of $g_{A B}, \gamma_{I J}$, and $b(x)$, and integrate over the extra dimensions 0 . From the metric ansatz (20) we have

$$
\begin{aligned}
\sqrt{-G} & =b^{n} \sqrt{-g} \sqrt{\gamma} \\
R\left[G_{a b}\right] & =R\left[g_{A B}\right]+b^{-2} R\left[\gamma_{i j}\right]-2 n b^{-1} g^{A C} \nabla_{A} \nabla_{C} b-n(n-1) b^{-2} g^{A C}\left(\nabla_{A} b\right)\left(\nabla_{C} b\right)
\end{aligned}
$$

where $\nabla_{A}$ is the covariant derivative associated with the $(N+1)$-dimensional metric $g_{A B}$. We denote by $\mathcal{V}$ the volume of the extra dimensions when $b=1$; it is given by

$$
\mathcal{V}=\int d^{n} y \sqrt{\gamma}
$$

The $(N+1)$-dimensional Newton's constant $G_{N+1}$ is related to its higher-dimensional version by

$$
\frac{1}{16 \pi G_{N+1}}=\frac{\mathcal{V}}{16 \pi G_{N+n+1}}
$$

We are thus left with

$$
\begin{aligned}
S=\int d^{N+1} x \sqrt{-g} & \left\{\frac { 1 } { 1 6 \pi G _ { N + 1 } } \left[b^{n} R\left[g_{A B}\right]-2 n b^{n-1} g^{A B} \nabla_{A} \nabla_{B} b\right.\right. \\
& \left.\left.-n(n-1) b^{n-2} g^{A B}\left(\nabla_{A} b\right)\left(\nabla_{B} b\right)+n(n-1) k b^{n-2}\right]+\mathcal{V} b^{n} \widehat{\mathcal{L}}_{M}\right\}
\end{aligned}
$$

where the curvature parameter $k$ of $\gamma_{i j}$ is given by (17).

\footnotetext{
${ }^{3}$ As is well known, if one substitutes a metric (or other field) ansatz into an action, there is a danger that variation of the new action will not reproduce all of the field equations, since the metric (or other field) variations are restricted by the ansatz. If the ansatz arises from symmetry assumptions, then, typically, the missing field equations will be automatically satisfied by virtue of the assumed symmetry. It is not difficult to verify that this is the case here, so that the reduced action that we will obtain below does indeed yield all of Einstein's equations. On the other hand, if the ansatz arises, e.g., from a gauge choice, then there is no reason to expect that the new action will reproduce all of the field equations. An example of this can be found in the last reference of [1], where the gauge choice $g_{00}=1$ is imposed in the action, and the action consequently fails to yield the 00-component of Einstein's equation.
} 
Through a change of variables and a conformal transformation,

$$
\begin{aligned}
& b(x)=e^{\beta(x)}, \\
& g_{A B}=e^{-2 n \beta /(N-1)} \tilde{g}_{A B},
\end{aligned}
$$

we can turn this reduced action into that of a scalar field coupled to gravity in the so-called "Einstein frame" (where the gravitational Lagrangian is simply the Ricci scalar, with no multiplicative scalar fields). Some algebra yields

$$
\begin{array}{r}
S=\int d^{N+1} x \sqrt{-\tilde{g}}\left\{\frac { 1 } { 1 6 \pi G _ { N + 1 } } \left[R\left[\tilde{g}_{A B}\right]-\frac{n(N+n-1)}{N-1} \tilde{g}^{A B}\left(\tilde{\nabla}_{A} \beta\right)\left(\tilde{\nabla}_{B} \beta\right)\right.\right. \\
\left.\left.+n(n-1) k e^{-2 \frac{N+n-1}{N-1} \beta}\right]+\mathcal{V} e^{-2 \frac{n}{N-1} \beta} \widehat{\mathcal{L}}_{M}\right\},
\end{array}
$$

where we have dropped terms that are total derivatives.

To turn $\beta$ into a canonically normalized scalar field, we make one final change of variables, to

$$
\phi=\sqrt{\frac{n(N+n-1)}{N-1}} M_{P} \beta,
$$

where the reduced Planck mass is $M_{P}^{-1}=\sqrt{8 \pi G_{N+1}}$ (in units where $\hbar=1$ ). We are then left with

$$
\begin{aligned}
S=\int d^{N+1} x \sqrt{-\tilde{g}} & \left\{\frac{1}{2} M_{P}^{2} R\left[\tilde{g}_{A B}\right]-\frac{1}{2} \tilde{g}^{A B}\left(\tilde{\nabla}_{A} \phi\right)\left(\tilde{\nabla}_{B} \phi\right)\right. \\
& \left.+\frac{1}{2} k n(n-1) M_{P}^{2} e^{-2 \sqrt{\frac{N+n-1}{n(N-1)}} \phi / M_{P}}+\mathcal{V} e^{-2 \sqrt{\frac{n}{(N-1)(N+n-1)}} \phi / M_{P}} \widehat{\mathcal{L}}_{M}\right\} .(31)
\end{aligned}
$$

Specializing to the case $n=2$ and $N=3$, we see that $\phi$ is a canonically normalized scalar with a potential

$$
V(\phi)=-k M_{P}^{2} e^{-2 \phi / M_{P}}-\mathcal{V} e^{-\phi / M_{P}} \widehat{\mathcal{L}}_{M}(\phi) .
$$

The stability properties and cosmological evolution of this model will evidently depend on the choice of matter Lagrangian $\widehat{\mathcal{L}}_{M}(\phi)$.

As an attempt at a realistic scenario, we take $\widehat{\mathcal{L}}_{M}$ to consist of a cosmological constant plus an abelian gauge field wrapped around the extra dimensions. In the six-dimensional picture, we have an antisymmetric field strength $F_{a b}$ which we take to be proportional to the volume form of the extra-dimensional metric $\gamma_{i j}$; in components we have

$$
F_{45}=-F_{54}=\sqrt{\gamma} F_{0}
$$


where $F_{0}$ is a constant and all other components vanish. In particular, the components of $F_{a b}$ are independent of $b$. (It is straightforward to check that such a field satisfies Maxwell's equations, either directly or by using conservation of flux.) We have a six-dimensional matter Lagrangian

$$
\widehat{\mathcal{L}}_{M}=-\lambda_{6}-\frac{1}{4} G^{a c} G^{b d} F_{a b} F_{c d},
$$

where $\lambda_{6}$ is the six-dimensional vacuum energy density. Upon dimensional reduction this yields

$$
\mathcal{V} \widehat{\mathcal{L}}_{M}=-\lambda_{4}-f_{0}^{2} e^{-2 \phi / M_{P}}
$$

where $\lambda_{4}=\mathcal{V} \lambda_{6}$ is the "bare" four-dimensional vacuum energy density and $f_{0}^{2}=\frac{1}{2} \mathcal{V} F_{0}^{2}$ is a (nonnegative) constant.

The effective potential for $\phi$ in this model is therefore

$$
V(\phi)=-k M_{P}^{2} e^{-2 \phi / M_{P}}+\lambda_{4} e^{-\phi / M_{P}}+f_{0}^{2} e^{-3 \phi / M_{P}}
$$

We would like to choose the parameters $\lambda_{4}$ and $f_{0}$ such that there is a local minimum of the potential for which $V=0$ (so that the effective four-dimensional cosmological constant vanishes and we can have a flat-spacetime solution). It is clear from (36) that choosing $k \leq 0$ cannot lead to an isolated minimum at finite $\phi$, in accordance with the results of the previous section. Instead we choose $k>0$ and

$$
\lambda_{4}=f_{0}^{2}=\frac{1}{2} k M_{P}^{2}
$$

There is then a local minimum at $\phi=0$, and the potential takes the form shown in Figure 1; the mass of the radion is

$$
m_{\phi}^{2}=\left.\frac{d^{2} V}{d \phi^{2}}\right|_{\phi=0}=k
$$

Note that there is also a minimum as $\phi$ goes to infinity, corresponding to blowing up the size of the extra dimensions. This raises the possibility of a Brustein-Steinhardt problem [19], if cosmological evolution tends to overshoot the desired minimum at $\phi=0$. However, this does not seem like a serious obstacle to the most popular scenarios, which feature two extra dimensions, a six-dimensional Planck scale of order $1 \mathrm{TeV}$, and a volume $\mathcal{V}$ of order $(1 \mathrm{~mm})^{2} \sim\left(10^{-3} \mathrm{eV}\right)^{-2}$, so that the four-dimensional Planck scale is $M_{P} \sim 10^{18} \mathrm{GeV}$ [1]. From (38) the radion is a light field, with mass set by the length scale of the extra dimensions. 


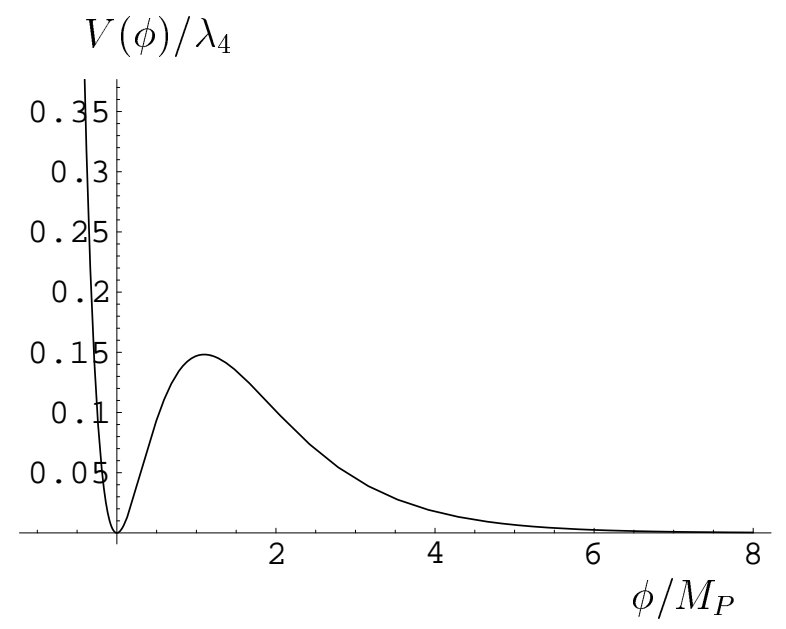

Figure 1: The effective potential (36) of the radion in the model described in the text.

Meanwhile, the height of the barrier in Figure 1 is of order $V\left(\phi=M_{P}\right) \sim k M_{P}^{2} \sim 1 \mathrm{TeV}^{4}$. Thus, in order for the field to roll coherently over the barrier, it would have to have an energy density of order the (six-dimensional) scale of quantum gravity in this model, which is in a regime in which our classical analysis has no right to be applicable. However, another potential difficulty with this model arises from the possibility that perturbations in the early universe could lead to localized regions in which $\phi$ tunnels over the barrier, either due to thermal or quantum fluctuations, giving rise to "bubbles" in which the extra dimensions expand to infinity. We do not analyze this issue here.

Thus, with this model, the effect of the extra dimensions is to introduce an additional massive scalar field (the radion) into the four-dimensional theory. It is therefore straightforward to answer questions concerning stability; if we consider a solution in which the radion is slightly perturbed from its equilibrium value, it will act as an energy source in the large dimensions, which will evolve as a Friedmann-Robertson-Walker spacetime. This spacetime will have a Big-Bang singularity, but is nevertheless cosmologically acceptable. The radion acts as a massive scalar which contributes to the total energy density of the universe; so long as its contribution is not too large (or it can decay away), it will not be cosmologically harmful. 


\section{Stabilization vs. minimization of energy}

When one is attempting to stabilize the extra dimensions by adding some form of matter, it might appear natural to expect that the extra dimensions will be stable when the total matter energy is minimized (the total matter energy being the integral of the energy density of matter over the volume). Indeed, this conclusion is rigorously correct for systems normally considered in non-gravitational physics: the total energy of matter normally is positive definite (thereby providing an appropriate norm) and conserved, and only positive energy can be radiated to infinity. Consequently a small perturbation about the state of minimum energy cannot drive the system far from this state. However, this argument fails in general relativity, since there is no global conservation law for the total energy of matterf. The main purpose of this section is to consider a simple cosmological model illustrating how one can be led seriously astray by non-gravitational intuition when determining the stability of spacetimes in general relativity.

Consider an $(n+1)$-dimensional spacetime of constant spatial curvature $k$, so that the metric takes the conventional Robertson-Walker form

$$
d s^{2}=-d t^{2}+a(t)^{2} \gamma_{\alpha \beta} d x^{\alpha} d x^{\beta}
$$

This space is to be filled with a uniform, perfect fluid of energy density $\rho$, whose pressure, $p$, is a function of $\rho$. By conservation of stress-energy, we have

$$
p=-\rho-\frac{a}{n} \frac{d \rho}{d a}
$$

This equation can be integrated to express $\rho$ (and, hence, $p$ ) in terms of $a$, and, in the following, it will be convenient to view $\rho$ and $p$ as functions of $a$. The quantity $U=\rho a^{n}$ represents the "total energy" (per comoving volume element) of the fluid. Minimizing $U$ with respect to $a$, we obtain

$$
\frac{d U}{d a}=-p n a^{n-1}
$$

Thus for an extremum of $U$ we must have $p=0$. Furthermore, for this extremum to be a minimum, we require $d^{2} U / d a^{2}>0$, giving

$$
\left.\frac{d^{2} U}{d a^{2}}\right|_{p=0}=-n a^{n-1} \frac{d p}{d a}>0,
$$

\footnotetext{
${ }^{4}$ Inflation provides a dramatic illustration of this fact.
} 
which of course is just the condition that $d p / d a$ be negative. Thus, analysis of the total matter energy in the manner normally done in non-gravitational physics suggests that space can be in equilibrium at some size only when the pressure is zero, and stable only if the pressure increases as the volume shrinks ("the more the system is squeezed, the harder it pushes back").

But now consider the analysis of equilibrium and stability in general relativity, as determined by Einstein's equation. For the Robertson-Walker metric (39) we obtain the Friedmann equations in $(n+1)$ dimensions,

$$
\begin{aligned}
\frac{1}{2} n(n-1)\left(\frac{\dot{a}}{a}\right)^{2} & =8 \pi G \rho-\frac{1}{2} n(n-1) k, \\
(n-1)\left(\frac{\ddot{a}}{a}\right)+\frac{1}{2}(n-1)(n-2)\left(\frac{\dot{a}}{a}\right)^{2} & =-8 \pi G p-(n-1)\left(\frac{n}{2}-1\right) k,
\end{aligned}
$$

where $k$ is the curvature parameter (7) for the spatial manifold. The conditions for equilib$\operatorname{rium}(\dot{a}=\ddot{a}=0$ and setting $a=1)$ are

$$
\begin{aligned}
\rho & =\frac{1}{16 \pi G} n(n-1) k, \\
p & =-\frac{n-2}{n} \rho .
\end{aligned}
$$

which bear little relationship to the condition $p=0$ obtained by extremization of total matter energy.

To determine whether the equilibrium solution is stable we examine the linearized equations of motion. We expand all variables to first order: $a=1+\delta a, \rho=\bar{\rho}+\delta \rho$, and $p=\bar{p}+\delta p$. Then

$$
\begin{aligned}
0 & =8 \pi G \delta \rho+n(n-1) k \delta a \\
(n-1) \ddot{\delta} a & =-8 \pi G \delta p+(n-1)(n-2) k \delta a \\
& =-8 \pi G \frac{d p}{d a} \delta a+(n-1)(n-2) k \delta a .
\end{aligned}
$$

To obtain a stark contradiction to the non-gravitational analysis, set $n=2$. The second of the equations for equilibrium (46) then reduces to $p=0$, which is precisely what was obtained by energy extremization. Therefore, we may compare the general relativistic conditions for stability with those that would be predicted by energy minimization. The second of the equations for stability (48) gives

$$
\ddot{\delta a}=-8 \pi G \frac{d p}{d a} \delta a
$$


In other words, this $(2+1)$-dimensional spacetime is stable only when $d p / d a$ is positive, exactly the opposite of eq. (42). Thus, in this model, the conditions for stable equilibrium in general relativity are that the total energy of matter be a maximum.

\section{Solutions with multiple compact spaces}

In this section, we generalize the discussion of sections 2 and 3 to the case of multiple maximally symmetric $n_{i}$-dimensional spaces. We begin by characterizing static solutions as in section 2. We then recast the full gravitational action as the action for a set of scalar fields evolving in a four-dimensional spacetime as in section 3, and discuss the possibility of stable solutions. Multiple compact spaces have been previously considered in [10, 13]; to this discussion we add an argument that the extra dimensions should be positively curved, and we consider the height of the barrier separating the static solution from one in which the scale factor for the extra dimensions runs away to infinity.

Our consideration of static spacetimes is a straightforward generalization of that in section 2 , so we will be concise. Consider a spacetime with spatial sections described by products of $m$ maximally symmetric manifolds, such that the metric may be written

$$
d s^{2}=-d t^{2}+\sum_{i=1}^{m} \gamma_{\alpha_{i} \beta_{i}}^{(i)} d x^{\alpha_{i}} d x^{\beta_{i}}
$$

where the $\gamma_{\alpha_{i} \beta_{i}}^{(i)}$ 's are metrics of constant curvature. The indices $\left\{\alpha_{i}, \beta_{i}\right\}$ run from $n_{i-1}+1$ to $n_{i}$ with $n_{0}=0$, i.e. the set of indices "runs over the submanifold $\mathcal{M}_{i}$ ". The curvature parameters $k_{i}$ are defined analogously to (7). For a stress-energy source, we consider a perfect fluid defined by an energy density $\rho$ and a pressure $p_{i}$ in each set of dimensions:

$$
\begin{gathered}
T_{00}=\rho \\
T_{\alpha_{i} \beta_{i}}=p_{i} \gamma_{\alpha_{i} \beta_{i}}^{(i)} .
\end{gathered}
$$

There are $m+1$ independent components of Einstein's equation: the 00 component,

$$
\sum_{j} \frac{1}{2} n_{j}\left(n_{j}-1\right) k_{j}=8 \pi G \bar{\rho}
$$

and the $\alpha_{i} \beta_{i}$ components,

$$
-\left(n_{i}-1\right) k_{i}+\sum_{j} \frac{1}{2} n_{j}\left(n_{j}-1\right) k_{j}=-8 \pi G \bar{p}_{i},
$$


where once again overbars remind us that we are considering static solutions, and $G$ is Newton's constant in the full spacetime.

The null energy condition implies

$$
\rho+p_{i} \geq 0
$$

while the conditions (5354) lead to the relation

$$
8 \pi G\left(\bar{\rho}+\bar{p}_{i}\right)=\left(n_{i}-1\right) k_{i}
$$

Thus, the left hand side of (56) is nonnegative, and there are no static solutions with any $k_{i}<0$ that obey the NEC, in accordance with the general argument given at the end of section 2. None of the spatial factors can be negatively curved; it is not possible to compensate for some negative curvature in one factor with additional positive curvature somewhere else. As before, if all of the curvature parameters vanish, we recover empty Minkowski space. The only static nonempty solutions will involve no negatively curved spaces and at least one positively curved space.

With the criteria for static solutions in hand, we will now explore the stability of these solutions to small perturbations in the scale factors. Following section 3, we first consider the conventional dimensional reduction of this theory, rewriting the $4+\Sigma n_{i}$ dimensional Lagrangian as a four dimensional Lagrangian plus $M$ scalar fields representing the size of each set of extra dimensions. (The sum is only over the extra dimensions, and $M$ refers to the number of sets of extra dimensions.)

Let $G_{a b}$ be the metric for the full spacetime with coordinates $X^{a}$, and denote by $G_{4+\mathcal{N}}$ Newton's constant in the full spacetime where $\mathcal{N}=\Sigma n_{i}$, the number of extra dimensions. The indices $a, b$ run from 0 to $3+\mathcal{N}$. We consider metrics of the form

$$
d s^{2}=G_{a b} d x^{a} d x^{b}=g_{A B} d x^{A} d x^{B}+\sum_{i=1}^{M} b_{i}^{2}(x) \gamma_{\alpha_{i} \beta_{i}}^{(i)}(y) d y^{\alpha_{i}} d y^{\beta_{i}}
$$

where $A$ and $B$ run over the four indices of the $3+1$ dimensional spacetime and each set $\left\{\alpha_{i}, \beta_{i}\right\}$ run over the appropriate submanifold. The action is as before:

$$
S=\int d^{4+\mathcal{N}} X \sqrt{-G}\left\{\frac{1}{16 \pi G_{4+\mathcal{N}}} R\left[G_{a b}\right]+\widehat{\mathcal{L}}_{M}\right\} .
$$


From the metric ansatz (57) we have

$$
\begin{aligned}
\sqrt{-G}= & \sqrt{-g} \prod_{i} b_{i}^{n_{i}} \sqrt{\gamma^{(i)}} \\
R\left[G_{a b}\right]= & R\left[g_{A B}\right]+\sum_{i=1}^{M}\left[b_{i}^{-2} R\left[\gamma^{(i)}\right]-2 n_{i} b_{i}^{-1} g^{A C} \nabla_{A} \nabla_{C} b_{i}\right. \\
& \left.\quad-n_{i}\left(n_{i}-1\right) b_{i}^{-2} g^{A C}\left(\partial_{A} b_{i}\right)\left(\partial_{C} b_{i}\right)-\sum_{j \neq i} n_{i} n_{j} b_{i}^{-1} b_{j}^{-1} g^{A C} \partial_{A} b_{i} \partial_{C} b_{j}\right],
\end{aligned}
$$

where $\nabla_{A}$ is the covariant derivative associated with $g_{A B}$. The volume of the extra dimensions when all $b_{i}=1$ is given by

$$
\mathcal{V}=\prod_{i} \int d^{n_{i}} y \sqrt{\gamma^{(i)}},
$$

and the four-dimensional Newton's constant $G_{4}$ is related to its higher-dimensional version by

$$
\frac{1}{16 \pi G_{4}}=\frac{\mathcal{V}}{16 \pi G_{4+\mathcal{N}}}
$$

We are thus left with

$$
\begin{aligned}
S=\int d^{4} x \sqrt{-g} & \left\{\frac { \prod _ { i } b _ { i } ^ { n _ { i } } } { 1 6 \pi G _ { 4 } } \left[R\left[g_{A B}\right]+\sum_{i=1}^{M}\left[n_{i}\left(n_{i}-1\right) k_{i} b_{i}^{-2}-2 n_{i} b_{i}^{-1} g^{A C} \nabla_{A} \nabla_{C} b_{i}\right.\right.\right. \\
& \left.\left.-n_{i}\left(n_{i}-1\right) b_{i}^{-2} g^{A C}\left(\partial_{A} b_{i}\right)\left(\partial_{C} b_{i}\right)-\sum_{j \neq i} n_{i} n_{j} b_{i}^{-1} b_{j}^{-1} g^{A C} \partial_{A} b_{i} \partial_{C} b_{j}\right]\right] \\
& \left.+\mathcal{V} \widehat{\mathcal{L}}_{M}\right\}
\end{aligned}
$$

where we have introduced the curvature parameter $k_{i}$ of each set of extra dimensions via $R\left[\gamma^{(i)}\right]=n_{i}\left(n_{i}-1\right) k_{i}$.

Through a change of variables and a conformal transformation,

$$
\begin{aligned}
\alpha_{i}(x) & =\ln b_{i}(x)-\lambda \\
g_{a b} & =e^{2 \lambda} \tilde{g}_{a b},
\end{aligned}
$$

where $\lambda=-\frac{1}{2} \sum n_{i} \ln b_{i}$, we can turn this reduced action into that of $M$ scalar fields coupled to gravity in the Einstein frame. Ignoring a total divergence term, after some algebra the reduced action becomes

$$
\begin{aligned}
S=\int \sqrt{-\tilde{g}}\{ & \frac{1}{16 \pi G_{4}}\left[\tilde{R}\left[\tilde{g}_{A B}\right]+\sum_{i} e^{-2 \alpha_{i}} n_{i}\left(n_{i}-1\right) k_{i}\right. \\
& \left.\left.+\sum_{i, j}\left(\eta_{i j} \tilde{g}^{A B} \tilde{\nabla}_{A} \alpha_{i} \tilde{\nabla}_{B} \alpha_{j}\right)\right]+\mathcal{V} e^{-\frac{2}{2+\mathcal{N}} \Sigma n_{i} \alpha_{i}} \mathcal{L}_{M}\right\},
\end{aligned}
$$


where

$$
\eta_{i j}=n_{i} n_{j} /(2+\mathcal{N})-n_{i} \delta_{i j}
$$

In order to have terms that look like the canonical kinetic terms for a set of scalar fields, we must diagonalize the quadratic form $\eta_{i j}$; this has been performed explicitly by [10, 13]. Thus, we may rewrite the action as

$$
\begin{aligned}
S=\int \sqrt{-\tilde{g}}\left\{\frac { 1 } { 1 6 \pi G _ { 4 } } \left[\tilde{R}\left[\tilde{g}_{A B}\right]+\sum_{i} e^{-2 \alpha_{i}} n_{i}\left(n_{i}-1\right) k_{i}\right.\right. \\
\left.\left.+\sum_{i}\left(\eta_{i}^{\prime} \tilde{g}^{A B} \tilde{\nabla}_{A}\left(\alpha_{i}^{\prime}\right) \tilde{\nabla}_{B}\left(\alpha_{i}^{\prime}\right)\right)\right]+\mathcal{V} e^{-\frac{2}{2+\mathcal{N}} \Sigma n^{\prime}{ }_{i} \alpha_{i}^{\prime}} \mathcal{L}_{M}\right\}
\end{aligned}
$$

where $\eta_{i}^{\prime}$ are the eigenvalues of $\eta_{i j}$ and the $\alpha_{i}^{\prime}$ are combinations of the $\alpha_{i}$ that correspond to the $\eta_{i}^{\prime}$. Since each $\alpha_{i}$ may be written as a linear combination of the $\alpha_{i}^{\prime}$, we have an action for $M$ scalar fields on a background spacetime. The potential for these fields is positive for $\mathcal{L}_{M}>0$, although from this form it is not clear in general whether the static point is stable.

As an example, consider the spacetime $\mathcal{M} \times S^{2} \times S^{2}$, where $\mathcal{M}$ is a $3+1$ dimensional homogeneous, isotropic model [10]. The action for this spacetime reduces to

$$
\begin{aligned}
S=\int \sqrt{-g} & \left(\frac{1}{2} M_{P}^{2} R\left[g_{A B}\right]-\frac{1}{2} g^{A B} \partial_{A} \phi \partial_{B} \phi-\frac{1}{2} g^{A B} \partial_{A} \psi \partial_{B} \psi\right. \\
& \left.+e^{-(\sqrt{3} \phi+\psi) / M_{P}} M_{P}^{2} k_{f}+e^{-(\sqrt{3} \phi-\psi) / M_{P}} M_{P}^{2} k_{h}-\mathcal{V} e^{-\frac{2}{\sqrt{3}} \phi / M_{P}} \mathcal{L}_{M}\right)
\end{aligned}
$$

where $\mathcal{V}$ is the volume of the extra dimensions as defined in eq. (61) above, and we have introduced

$$
\begin{aligned}
\phi & =\frac{1}{\sqrt{3}}\left(\alpha_{f}+\alpha_{h}\right) M_{P} \\
\psi & =\left(\alpha_{f}-\alpha_{h}\right) M_{P},
\end{aligned}
$$

where $f$ and $h$ label the spheres.

We choose the matter for this model to consist of a cosmological constant, $\lambda_{8}$, and abelian gauge fields, $F_{a b}$ and $H_{a b}$, so the matter Lagrangian is

$$
\mathcal{L}_{M}=-\lambda_{8}-\frac{1}{4} G^{a c} G^{b d} F_{a b} F_{c d}-\frac{1}{4} G^{a c} G^{b d} H_{a b} H_{c d}
$$

We take the gauge fields to wrap around each $S^{2}$, so that, in components, we have

$$
\begin{aligned}
& F_{45}=-F_{54}=\sqrt{\gamma^{(f)}} F_{0} \\
& H_{67}=-H_{76}=\sqrt{\gamma^{(h)}} H_{0},
\end{aligned}
$$


where $F_{0}$ and $H_{0}$ are constants, and all other components vanish. The conditions for a static solution are [see eqs. (53,56)]

$$
\begin{aligned}
8 \pi G_{8} \bar{\rho} & =3 K+k_{f}+k_{h} \\
8 \pi G_{8}(\bar{\rho}+\bar{P}) & =2 K \\
8 \pi G_{8}\left(\bar{\rho}+\overline{p_{f}}\right) & =k_{f} \\
8 \pi G_{8}\left(\bar{\rho}+\overline{p_{h}}\right) & =k_{h},
\end{aligned}
$$

where $G_{8}$ is the 8-dimensional Newton's constant, $K$ is the curvature parameter for the large dimensions, and $k_{f}$ and $k_{h}$ are the curvature parameters for $\gamma_{i j}^{(f)}$ and $\gamma_{i j}^{(h)}$, respectively. Consequently, we find that static solutions exist, provided that

$$
\begin{aligned}
\lambda_{4} & =\frac{1}{2} M_{P}^{2}\left(k_{f}+k_{h}\right) \\
K & =0 \\
f_{0}^{2} & =\frac{1}{2} M_{P}^{2} k_{f} \\
h_{0}^{2} & =\frac{1}{2} M_{P}^{2} k_{h},
\end{aligned}
$$

where

$$
\begin{aligned}
f_{0}^{2} & =\frac{1}{2} \mathcal{V} F_{0}^{2} \\
h_{0}^{2} & =\frac{1}{2} \mathcal{V} H_{0}^{2} .
\end{aligned}
$$

Thus, there exists a 2-parameter family of static solutions. It is convenient to choose as independent parameters the higher-dimensional (reduced) Planck mass and the ratio of the static curvature parameters of the two-spheres:

$$
\begin{aligned}
M_{8}^{-6} & =\sqrt{8 \pi G_{8}} \\
r & =\frac{k_{f}}{k_{h}} .
\end{aligned}
$$

In terms of these, the equilibrium volume of the internal space is set by

$$
\mathcal{V}=\frac{M_{P}^{2}}{M_{8}^{6}},
$$


where $M_{P}=10^{18} \mathrm{GeV}$ is the (reduced) four-dimensional Planck scale. We will assume that $M_{8}=1 \mathrm{TeV}$; this implies $\mathcal{V} \approx(30 \mathrm{keV})^{-4}$. The curvature parameters for the spheres can be determined from (86) and

$$
k_{f} k_{h}=\frac{16 \pi^{2}}{\mathcal{V}}
$$

we have for example

$$
k_{f}=4 \pi \frac{M_{8}^{3}}{M_{P}} \sqrt{r} .
$$

The potential in this matter model is

$$
\begin{gathered}
V(\phi, \psi)=2 \pi M_{P} M_{8}^{3}\left[\left(\frac{1+r}{\sqrt{r}}\right) e^{-\left(2 / \sqrt{3} M_{P}\right) \phi}+\sqrt{r}\left(e^{-2[(2 / \sqrt{3}) \phi+\psi] / M_{P}}-2 e^{-(\sqrt{3} \phi+\psi) / M_{P}}\right)\right. \\
\left.+\frac{1}{\sqrt{r}}\left(e^{-2[(2 / \sqrt{3}) \phi-\psi] / M_{P}}-2 e^{-(\sqrt{3} \phi-\psi) / M_{P}}\right)\right] .
\end{gathered}
$$

The fields which diagonalize the mass matrix at the equilibrium solution $(\phi=0, \psi=0)$ are

$$
\begin{aligned}
\Phi & =\sqrt{\frac{2-\mu_{\Phi}^{2}}{\mu_{\Psi}^{2}-\mu_{\Phi}^{2}}}\left(\phi-\frac{2(r-1)}{\sqrt{3}(r+1)\left(2-\mu_{\Phi}^{2}\right)} \psi\right) \\
\Psi & =\sqrt{\frac{\mu_{\Psi}^{2}-2 / 3}{\mu_{\Psi}^{2}-\mu_{\Phi}^{2}}}\left(\frac{2(r-1)}{\sqrt{3}(r+1)\left(\mu_{\Psi}^{2}-2 / 3\right)} \phi+\psi\right),
\end{aligned}
$$

where the $\mu$ 's are normalized masses,

$$
\begin{aligned}
& \mu_{\Phi}^{2}=\frac{m_{\Phi}^{2} M_{P}^{2}}{\lambda_{4}}=\frac{4}{3}\left(1-\sqrt{1-\frac{3 r}{(1+r)^{2}}}\right) \\
& \mu_{\Psi}^{2}=\frac{m_{\Psi}^{2} M_{P}^{2}}{\lambda_{4}}=\frac{4}{3}\left(1-\sqrt{1+\frac{3 r}{(1+r)^{2}}}\right)
\end{aligned}
$$

and $m_{\Phi}$ and $m_{\Psi}$ are the actual masses of the $\Phi$ and $\Psi$ fields respectively.

It is clear that replacing $r \rightarrow 1 / r$ is equivalent to interchanging the roles of the $F$ and $H$ gauge fields; we therefore only consider $r \geq 1$, or $k_{f} \geq k_{h}$ (so that the sphere with $F$ flux is smaller than that with $H$-flux). There is also an upper limit on $r$, derived from the requirement that the curvature of the extra dimensions not exceed the higher-dimensional Planck scale, $k_{f} \leq M_{8}^{2}$. From (89), setting $M_{8}=1 \mathrm{TeV}$ then implies

$$
r \leq 10^{28}
$$


There is a complementary constraint, that the larger two-sphere not be big enough that deviations from Newtonian gravity would have been detected in laboratory experiments; this corresponds to $k_{h}<\left(10^{-2} \mathrm{eV}\right)^{-2}[3]$. This bound is just saturated when $r=10^{28}$, so these bounds are essentially equivalent.

For finite values of $r$, the masses of both $\Phi$ and $\Psi$ are positive, implying stability of the system around the equilibrium solution. We would like to comment briefly on the cosmological acceptability of such a scenario, following the discussion of the height of the potential barrier investigated in section 3 . For a given value of $r$, the relevant height is that of the lowest barrier separating the equilibrium solution from infinity. This will correspond to a saddle point in $V(\Phi, \Psi)$, and the barrier height will be the value of $V(\Phi, \Psi)$ evaluated at the saddle point. Numerically investigating the potential (90) with $M_{8}=1 \mathrm{TeV}$, we find that the height of the barrier varies with $r$, but the barrier always remains at least $1 \mathrm{TeV}^{4}$. Since this is the fundamental scale of quantum gravity, we again find that there is no Brustein-Steinhardt problem [19] for this theory. At energy scales where classical gravity should be applicable, the field will never be able to classically roll over the barrier.

\section{Acknowledgments}

We thank Mark Trodden for helpful conversations. This work was supported in part by the NSF grants PHY 95-14726, PHY 01-14422, and PHY 00-90138, and DOE grant DE-FG0290ER-40560 to the University of Chicago, the Alfred P. Sloan Foundation, and the David and Lucile Packard Foundation. One of us (R.M.W.) would like to thank the Yukawa Institute of Kyoto University for its hospitality.

\section{References}

[1] N. Arkani-Hamed, S. Dimopoulos and G. Dvali, Phys. Lett. B 429, 263 (1998) hepph/9803315]; I. Antoniadis, N. Arkani-Hamed, S. Dimopoulos and G. Dvali, Phys. Lett. B 436, 257 (1998) [hep-ph/9804398]; N. Arkani-Hamed, S. Dimopoulos and J. MarchRussell, Phys. Rev. D 63, 064020 (2001) hep-th/9809124.

[2] V. A. Rubakov, hep-ph/0104152. 
[3] C. D. Hoyle, U. Schmidt, B. R. Heckel, E. G. Adelberger, J. H. Gundlach, D. J. Kapner and H. E. Swanson, Phys. Rev. Lett. 86, 1418 (2001) [hep-ph/0011014].

[4] W. D. Goldberger and M. B. Wise, Phys. Rev. Lett 834922 (1999) hep-ph/9907447.

[5] R. M. Wald: "General Relativity," The University of Chicago Press, Chicago (1984).

[6] T. Kaluza, Sitzungsber. Preuss. Akad. Wiss. Berlin (Math. Phys. ) K1, 966 (1921); O. Klein, Z. Phys. 37, 895 (1926) [Surveys High Energ. Phys. 5, 241 (1926)]; O. Klein, Nature 118, 516 (1926).

[7] E. Cremmer and J. Scherk, Nucl. Phys. B 108, 409 (1976); Z. Horvath, L. Palla, E. Cremmer and J. Scherk, Nucl. Phys. B 127, 57 (1977).

[8] P. G. Freund and M. A. Rubin, Phys. Lett. B 97, 233 (1980).

[9] S. Randjbar-Daemi, A. Salam and J. Strathdee, Nucl. Phys. B 214, 491 (1983); Phys. Lett. B 132, 56 (1983); Phys. Lett. B 135, 388 (1984); Nucl. Phys. B 242, 447 (1984); Phys. Lett. B 124, 345 (1983); Erratum: ibid. B 144, 455 (1984).

[10] U. Gunther and A. Zhuk, Phys. Rev. D 56, 6391 (1997) gr-qc/9706050; U. Gunther and A. Zhuk, Phys. Rev. D 61, 124001 (2000) hep-ph/0002009; U. Gunther and A. Zhuk, Class. Quant. Grav. 18, 1441 (2001) [hep-ph/0006283.

[11] R. Sundrum, Phys. Rev. D 59, 085010 (1999) hep-ph/9807348].

[12] D. Sudarsky and R.M. Wald, Phys. Rev. D 46, 1453 (1992).

[13] V. D. Ivashchuk and V. N. Zhuk, Nuovo Cim. B 104, 575 (1989); M. Rainer and A. Zhuk, Phys. Rev. D 54, 6186 (1996) gr-qc/9608020.

[14] L. Randall and R. Sundrum, Phys. Rev. Lett. 83, 3370 (1999) hep-ph/9905221].

[15] A. G. Cohen and D. B. Kaplan, Phys. Lett. B 470, 52 (1999) hep-th/9910132; I. Olasagasti, Phys. Rev. D 63, 124016 (2001) hep-th/0101203.

[16] N. Kaloper, J. March-Russell, G. D. Starkman and M. Trodden, Phys. Rev. Lett. 85, 928 (2000) hep-ph/0002001. 
[17] C. B. Netterfield et al., astro-ph/0104460; C. Pryke et al., astro-ph/0104490; R. Stompor et al., astro-ph/0105062 (2001).

[18] J. M. Overduin and P. S. Wesson, Phys. Rep. 283303 (1997) gr-qc/9805018.

[19] R. Brustein and P. J. Steinhardt, Phys. Lett. B 302, 196 (1993) hep-th/9212049. 